\title{
EAOR: Energy Aware Optimized Routing Protocol in Mobile Ad Hoc Networks
}

\author{
F. Vinod Alexander B.Tech., M.E ${ }^{1}$, R. Vidhya M.Sc., M.Phil., M.E. ${ }^{2}$ \\ CSI College of Engineering, The Nilgiris ${ }^{1}$ \\ Assistant Professor, CSE Dept, CSI College of Engineering, The Nilgiris ${ }^{2}$
}

\begin{abstract}
Mobile Ad hoc Networks (MANETs) in terms of resources, robustness and efficiency for replicated service applications. Most of the anycast routing protocols select unstable and congested intermediate nodes, thereby causing frequent path failures and packet losses. We propose a mobility and quality of service aware routing scheme in MANETs that employs three models: node movement stability, channel congestion, and link/route expiry time. These models are used in the route discovery process to select nearest k-servers. A server among k-servers is selected based on less congestion, route expiry time, number of hops, and better stability. The simulation results indicate that proposed method demonstrates reduction in control overheads, path delays and improved packet delivery ratio compared to existing methods such as queue utilization, residual energy and node degree.
\end{abstract}

Keywords: Mobile Ad-Hoc Networks, Anycast routing, Energy Efficiency, Channel Congestion.

\section{INTRODUCTION}

Mobile Ad hoc Networks (MANETs) consists of a set of wireless mobile nodes communicating to each other without any centralized control or fixed network infrastructure and can be deployed quickly. The potential applications include emergency disaster relief, battlefield situations, mine site operations, and wireless classrooms or meeting rooms in which participants wish to share information or to acquire data. Anycast is an important way of communication for replicated service applications in terms of resources, robustness and efficiency, when mobility and link disconnections are frequent.

Anycast allows a source node to transmit packets to a single destination node out of set of several destination nodes. In previous framework, we used a novel solution to defend the OLSR protocol from node isolation attack by employing the same tactics used by the attack itself. Through extensive experimentation, we demonstrate that the proposed protection prevents more than 95 percent of attacks. Last, we suggest that this type of solution can be extended to other similar DOS attacks on OLSR. But It takes more energy for detecting malicious nodes. It increase time delay, low energy efficiency and high energy consumption. In proposed schemes.

The objective of this paper is to design and analyze stability and QoS based anycast routing scheme in MANET to improve the performance and enhance the service availability through the method of evenly distributed traffic load. The scheme uses Dynamic Source Routing (DSR) as basic route finding protocol along with stability and QoS models. Our contributions as compared to existing works are as follows. Designing a mathematical model for selecting stable nodes based on node's own stability, self stability, and neighbor nodes stability. Designing a mathematical model for selecting non congested nodes based on channel load and node buffer occupancy. Designing a mathematical model for finding link expiry time between pair of nodes. Design of route discovery process, which includes request phase to find routes to anycast servers through forwarding intermediate nodes which satisfy stability, congestion criteria and also meet the route expiry deadline; and designing route maintenance procedure to handle node and link failures. Simulator to perform the current method. part II explain the background information about the energy efficiency routing schemes. part III discuss about the new proposed method. Finally simulation and results are discussed in part IV.

\section{RELATED WORK}

Reducing energy consumption in mobile communications has attracted increasing attention recently. Advanced physical layer techniques such as multiple-input multiple-output (MIMO) and orthogonal frequency division multiplexing(OFDM), cognitive radio, network coding, cooperative communication, etc.; In this article, we overview these technologies and present the state-of-the-art on each aspect. Some challenges that need to be solved in the area are also described.[4],[14] Here, the joint power and subcarrier allocation problem is solved in the context of maximizing the energy-efficiency (EE) of a multi-user, multi-relay orthogonal frequency division multiple access 
(OFDMA) cellular network, where the objective function is formulated as the ratio of the spectral-efficiency (SE) over the total power dissipation. It is proven that the fractional programming problem considered is quasi-concave so that Dinkelbach's method may be employed for finding the optimal solution at a low complexity.

In this article, the cross-layer design problem of joint multiuser detection and power control is studied, using a gametheoretic approach that focuses on energy efficiency. The uplink of a direct-sequence code-division multiple-access data network is considered, and a non cooperative game is proposed in which users in the network are allowed to choose their uplink receivers as well as their transmit powers to maximize their own utilities. [8], [3] new concept called "reality check" and present a method of using a protocol model with reality check for wireless networks. it is possible to narrow the solution gap between the two models. Our simulation results confirm that this gap is indeed small (or even negligible). Thus, our methodology of joint reality check and interference range setting retains the protocol model as a viable approach to analyze multihop wireless networks. [2] we address this problem by considering a UWB-based ad hoc network. we formulate this utility maximization problem into a nonlinear programming (NLP) problem, which takes into consideration routing, scheduling, and power control. We develop a solution procedure based on the so-called branch-and-bound framework. [7] In this paper, energy efficient spectrum access is considered for a wireless cognitive radio ad hoc network, has limited energy, and the network is an OFDMA system operating on time slots. Given the data rate requirement and maximal power limit, a constrained optimization problem is formulated for each individual user to minimize the energy consumption per bit. [15] We analyze the total energy consumption of the proactive and the reactive networking strategies, taking into account transmitting, listening, and sleeping energy. Energy efficiency and overhead at the physical and the network layers are evaluated against message duty cycle, channel fading rate, and node mobility. The crossover point in message duty cycle below which reactive network has assured advantages is obtained. [6] This paper addresses link adaptive transmission for maximizing energy efficiency, as measured by the "throughput per Joule" metric. In contrast to the existing water-filling power allocation schemes that maximize throughput subject to a fixed overall transmit power constraint our scheme maximizes energy efficiency by adapting both overall transmit power and its allocation, according to the channel states and the circuit power consumed. We demonstrate the existence of a unique globally optimal link adaptation solution and develop iterative algorithms to obtain it. [16] multi input-single-output (MISO) is adopted to improve the energy efficiency of the network. We first establish the energy model of multihop cooperative MISO transmission. Based on the model, the energy consumption per bit of the network with high node density is minimized numerically by finding an optimal hop distance, and, to get the global minimum energy consumption, both hop distance and the number of cooperating nodes around each relay node for multihop transmission are jointly optimized. [1] Cognitive radio networks (CRNs) have the potential to utilize spectrum efficiently and are positioned to be the core technology for the next-generation multihop wireless networks. An important problem for such networks is its capacity. We study this problem for CRNs in the SINR model, which is considered to be a better characterization of interference (but also more difficult to analyze) than disk graph model. [12] We study resource allocation for energy-efficient communication in multi-cell orthogonal frequency division multiple access (OFDMA) downlink networks with cooperative base stations (BSs). We formulate the resource allocation problem for joint BS zero-forcing beamforming (ZFBF) transmission as a non-convex optimization problem which takes into account the circuit power consumption, the limited backhaul capacity, and the minimum required data rate.

\section{PROPOSED METHOD}

In our proposed work the scheme uses Dynamic Source Routing (DSR) as basic route finding protocol along with stability and QoS models. Our contributions as compared to existing works are as follows. Designing a mathematical model for selecting stable nodes (with respect to position) based on node's own stability, i.e., self-stability, and neighbor nodes stability. Designing a mathematical model for selecting non congested nodes based on channel load and node buffer occupancy. Designing a mathematical model for finding link expiry time between pair of nodes. Design of route discovery process, which includes request phase to find routes to anycast servers through forwarding intermediate nodes which satisfy stability, congestion criteria and also meet the route expiry deadline; and reply phase to update routing cache and confirm the routes found in request phase, and designing route maintenance procedure to handle node and link failures.

According to the literature survey, it has been observed that there is scope for improving anycast routing schemes in MANETs in terms of control overheads, load balancing, stable routes, and QoS. Thus there is a need to develop a robust and an efficient movement stability and QoS based congestion aware anycast routing scheme in MANET. We have designed three major models in our approach to anycast routing discovery problem. (1) Stability model to identify stable nodes, (2) congestion model to take QoS into consideration by checking congestion aware parameters like channel load, and buffer occupancy, and (3) link expiry time model to make sure the link duration will fall within an acceptable range. 


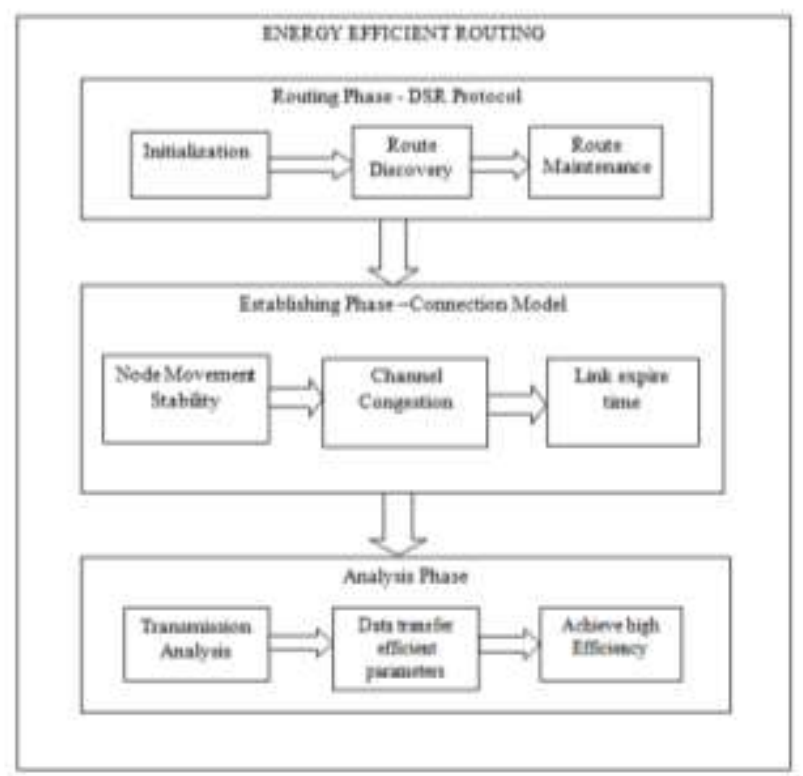

Fig.1. Proposed system design

\section{A. System Initialization \\ DSR Protocol}

Dynamic source routing (DSR) is an on-demand reactive routing protocol designed to restrict the bandwidth consumed by control packets, by eliminating the periodic table update messages required in the table driven proactive approach. It uses source routing instead of relying on the routing table at each intermediate node. DSR is beaconless and hence does not require periodic hello packet transmissions. Route construction phase establishes a route by flooding route request (RR) packets in the network. An intermediate node, upon receiving a RR packet, checks the sequence number on the packet before forwarding it .

\section{Route Discovery}

We modify Dynamic Source routing (DSR) by applying parameters supported by stability, congestion and route expiry models in route establishment phase. Routing considers the parameters at each node for route request propagation and path(s) finding between client and servers. It also uses routing information cache (RIC) at each node that facilitates route finding by providing path information from the existing database. RIC will reduce route request propagation overheads. This section presents databases, route request (RR) packets, route reply (RP) packets, route error (RE) packets, and RIC.

\section{Route Maintenance}

The packet format for anycast routing is almost similar to RR packet with few changes in RR packet. The changes in RR packet to convert it into RP packet are as follows: When RR packet reaches the server from the designated client, client address and server address are interchanged, RR packet contains the list of nodes along the route it has travelled (route record is reversed). RP packet from the server is sent to client on a route given in its route record. Next hop address will be picked from the route record at every hop. RE packet is generated when a node is unable to send the packets either due to link failure or congestion. Some of the fields of this packet are client address, server address, route record and sequence number. Whenever a node identifies link failure, it generates RE packet to either client or server. If link failure occurs in forward journey of a RR packet (from client to server), RE packet is sent to the client.

\section{B. Connection Model}

Node Stability Model

The stable nodes are necessary in forwarding group to provide better packet delivery services. Node stability in terms of movement around its current position gives us an idea of stationary property of node. Node stability metrics are used to identify stable nodes in a path for forwarding packets from a source to anycast node. Two metrics are identified to represent node movement stability as the quality of connectivity: self stability, and neighbor nodes stability. The steps in finding the stability of a node are as follows. (1) All the nodes in MANET find the self movement stability, i.e, node movement relative to its previous position, and (2) find neighbor node movement stability of all the nodes in MANET by considering the neighbors self stability. Each node in a MANET will compute the node movement stability factor based on self and neighbor nodes movement stability. 
Consider the scenario, where a node with transmission range ' $r$ ' moves from position ( $x r$;yr) to(xn;yn) in a given time window by a distance ' $d$ '. When a node moves from its previous position, its movement stability relative to previous position keeps varying, and the Distance of movement of a node (dti) in a time window ' $t$ ' can be measured by using given $\mathrm{Eq}$,

$$
d_{i}^{t}=\sqrt{\left(x_{n}-x_{r}\right)^{2}+\left(y_{n}-y_{r}\right)^{2}}
$$

Based on the movement of the distance at every time window, the self stability metric $(\mathrm{Ns}(\mathrm{t}))$ can be estimated as given in $\mathrm{Eq}(\mathrm{Ns}(\mathrm{t})$ ) varies between 0 and 1 . When the movement distance (dTi) of a node increases, the self stability value will decrease. For the requirement of the higher degree of movement stability, ' $r$ ' can be replaced by ' $r / 2$ ' or ' $r / 4$ ' or ' $r / 8$ ' etc.

$$
S_{5}(t)= \begin{cases}1-\frac{d}{r / 2} & \text { if } 0 \leqslant d_{i}^{t}<r / 2 \\ 0 & \text { Otherwise }\end{cases}
$$

There are some limitations in calculation of self-stability due to the influence of GPS accuracy and resolution. Better results can be estimated with higher accuracy and resolution in GPS. This work assumes that GPS accuracy and resolution is limited to $95 \%$ and $7.8 \mathrm{~m}$, respectively.

The degree of a node ' $n$ ' is represented as number of links (or nodes) connected to it, and is denoted as 'ND'. The neighbor node stability of a node $(\mathrm{Ns}(\mathrm{t}))$ with respect to neighbors at time ' $\mathrm{t}$ ' can be expressed as

$$
N_{s}(t)=\alpha \times \frac{1}{N D} \sum_{i=1}^{N D} S_{s, i}(t)+(1-\alpha) \times N_{s}(t-1)
$$

Node movement stability factor $\mathrm{Ns}(\mathrm{t})$ : It defines the stability of a node associated with self and neighbor node movement stability in a given time interval ' $t$ '. This can be expressed as

$$
\operatorname{Nsf}(t)=f\left(S_{s}(t), N_{s}(t)\right)=\beta S_{s}(t)+(1-\beta) N_{s}(t)
$$

In Ns(t), higher weightage is given to the first term, considering the fact that nodes are mobile and it is likely for a node to move with respect to its earlier position. Similarly, in Ns(t), higher weightage is given to first term. Stability factor of a node is computed only if self-stability and neighbor stability is greater than zero. Thus our scheme extracts the highly stable nodes and adjusts the network topology for routing restricted to stable nodes so as to reduce the probability of link failure.

\section{Channel Congestion}

To address the congestion problem in MANET, following two models are proposed: channel load model and buffer occupancy mode.

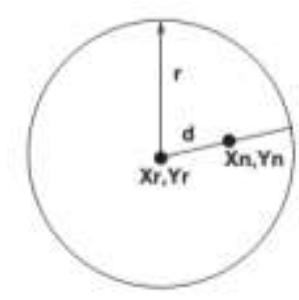

Channel load Model: Congestion in a network is measured based on rate of change in channel load (CL). This can be represented in $\mathrm{Eq}$ where $\mathrm{T}$ busy and $\mathrm{T}$ idle are busy and idle times of the channel measured in seconds in a given time window

$$
\mathrm{CL}=\frac{T_{\text {busy }}}{T_{\text {busy }}+T_{\text {idle }}}
$$

As channel load increases, congestion in the network increases. Thus change in rate of channel load in a particular time window indicates change in congestion level. The change in channel load with respect to time can be represented by means of slope as min dicating either rise or fall in the congestion level 


$$
\text { Ave_m }(t)=\frac{\sum_{i=1}^{n} m_{i}}{n}
$$

Based on the A vem, Channel Congestion Factor (CCF) in a time window $t$ can be interpreted by comparing it with allowed threshold value as decided by the administrator as given in Eq. The value of the $\mathrm{CCF}(\mathrm{t})$ varies between 0 (no congestion) and

$$
\operatorname{CCF}(t)= \begin{cases}\text { Avem } m(t) & \text { if } 0<\text { Ave } m(t)<\text { Threshold } \\ 1 & \text { if Ave.m }(t) \geqslant \text { Threshold } \\ 0 & \text { if Ave } m(t) \leqslant 0\end{cases}
$$

Link Buffer Occupancy: (LBO) Model: A node should maintain an average buffer level to avoid congestion and frequent link failures. LBO is defined as the ratio of queue occupancy (QOCC) by a link to maximum queue size (QSIZE) of the buffer in a given time window at intermediate node

$$
\mathrm{LBO}=\frac{\mathrm{QOCC}}{\mathrm{QSIZE}}
$$

The average of intermediate node's buffer occupancy (Ave_LBO) by all associated direct links of a node is given by

$$
\text { Ave_LBO }(t)=\frac{\sum_{i=1}^{n} \mathrm{LBO}_{i}}{n}
$$

As similar to $\mathrm{CCF}(\mathrm{t})$, we estimate the Buffer Congestion Factor $(\mathrm{BCF}(\mathrm{t}))$ based on buffer occupancy in a given time window t. Our routing scheme uses $\mathrm{CCF}(\mathrm{t})$ and $\mathrm{BCF}(\mathrm{t})$ and computes link congestion factor $(\mathrm{LCF})$ for QoS based applications to find route from a client to the server. It indicates the congestion of the direct link between intermediate nodes. $\mathrm{LCF}(\mathrm{t})$ (a normalized value) given in Eq helps in selecting non congested nodes with sufficient buffer capacity for routing in anycast networks. The smoothed value of $\mathrm{LCF}(\mathrm{t})$ denoted as $\mathrm{CF}(\mathrm{t})$ is given in Eq. The weight factors denote the relative importance of the quantities of LCF in current and previous time windows.

$$
\begin{aligned}
& \operatorname{LCF}(t)=\frac{\operatorname{CCF}(t)+\operatorname{BCF}(t)}{2} \\
& \operatorname{CF}(t)=\operatorname{LCF}(t-1) \times w+(1-w) \times \operatorname{LCF}(t)
\end{aligned}
$$

Lower value of $\mathrm{CF}(\mathrm{t})$ denotes lesser congestion at a node.

Link expire time : Link Expiration Time (LET) Model to reduce the data packets loss due to link failures. LET defines the lifetime of a link between intermediate nodes. In most existing protocols, a mobile host will keep using the route until the link is broken. In this model, we use a proactive method of finding the duration of time that two neighbors remain connected if the speed, direction, and radio propagation range are known. Predicting the LET along each hop on the route will facilitate the prediction of Route Expiration Time (RET). RET is defined as life time of route between client and server.

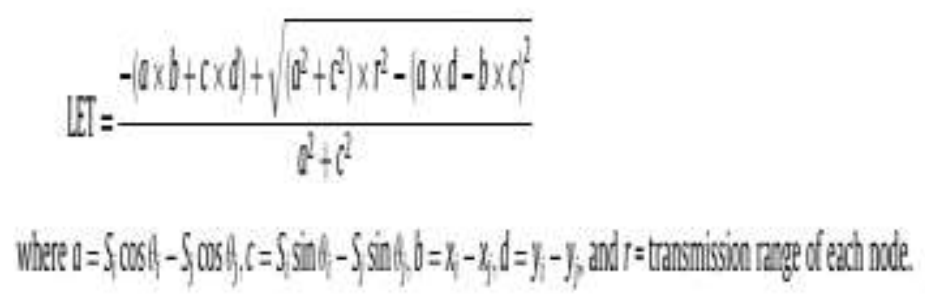

Anycast QoS based path creation involves two phases; a request phase and a reply phase. Request phase invokes route discovery process to find routes to anycast servers through stable and non-congestion intermediate nodes. Reply phase involves updating of RIC and conforming the routes found in request phase. Stable nodes are the one who satisfy stability criteria and non-congestion requirement of an application, based on our stability and congestion models. 


\section{Analysis Phase}

We have simulated proposed scheme for various network scenarios using $\mathrm{C}$ programming language. Simulation environment for the proposed work consists of four models: (1) Network model, (2) Channel model, (3) Mobility model, and (4) Traffic model.

Network Model: An ad hoc network is generated in a given area. It consists of several number of mobile nodes that are placed randomly within a given area. The coverage area around each node has a limited bandwidth that is shared among it's neighbor.

Channel Model: It assumes the free space propagation model and error free channel. To access the channel, ad hoc nodes use CSMA/CA (Carrier Sense Multiple Access with Collision Avoidance) media access protocol to avoid possible collisions and subsequent packet drops.

Mobility Model: We use a random way-point (RWP) mobility model based upon three parameters; speed of movement, direction for mobility and time of mobility. In RWP, each node picks a random destination uniformly within an underlying physical space, and travels with a given speed.

Traffic Model: It is a constant bit rate model that transmits a certain number of fixed size packets in a flow. Following metrics have been used to analyze the performance. (1) Packet Delivery Ratio (PDR): It is the ratio of number of average data packets received at anycast server to the number of data packets sent by the client. (2) Packet Overhead: It measures the ratio of control packets (RR, RP and hello packets) sent to the network to the total number of average data packets delivered to the server and (3) Average end-to-end delay: It is the average delay experienced by the successfully delivered packets in reaching the server.

\section{RESULTS AND DISCUSSION}

We use Network simulator version-2 (NS2) to show the performance of our proposed scheme. A wireless ad hoc network consists of mobile nodes are randomly deployed in this simulation. DSR protocol is used. We compared to existing scheme, our proposed scheme has better performance in terms of energy consumption, Delay, PDR and throughput. The following section shows the simulation parameters, results and comparison performance of the proposed system.

\section{Performance Results}

In this section, the performance of our protocol is compared with the existing method in terms of Delay, energy consumption, packet delivery ratio and throughput.

Delay

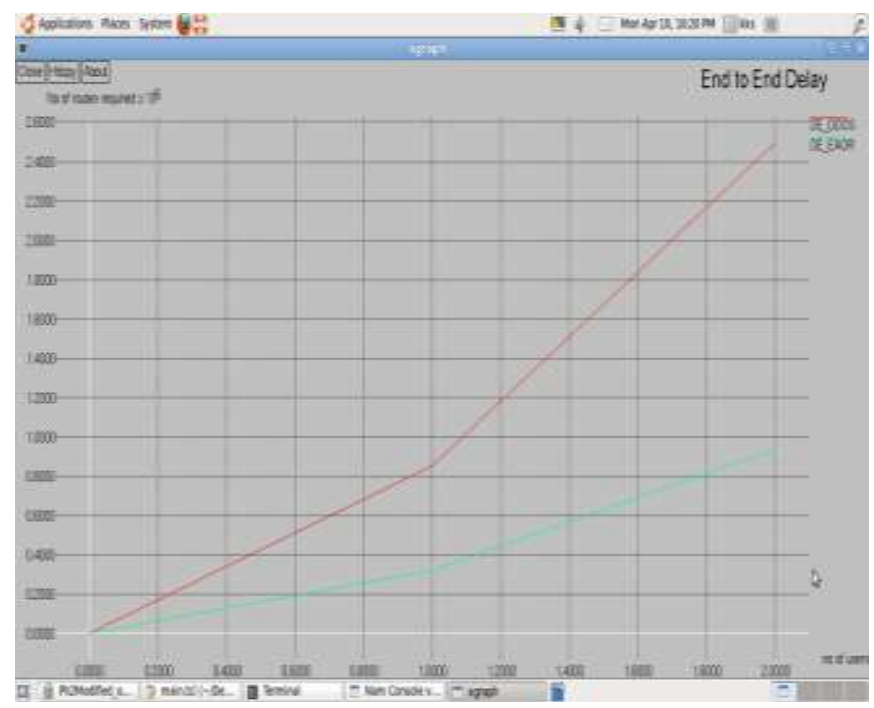

Above graph shows the comparison of existing and proposed scheme in terms of Delay. In this figure, the performance of proposed management scheme is reduced delay ratio level as compared to existing scheme.

ThroughputRatio

Bellow graph shows the comparison of existing and proposed scheme in terms of Throughput. In this figure, the performance of proposed scheme is good Throughput level as compared to existing scheme. 


\section{IJARCCE}

Vol. 6, Issue 4, April 2017

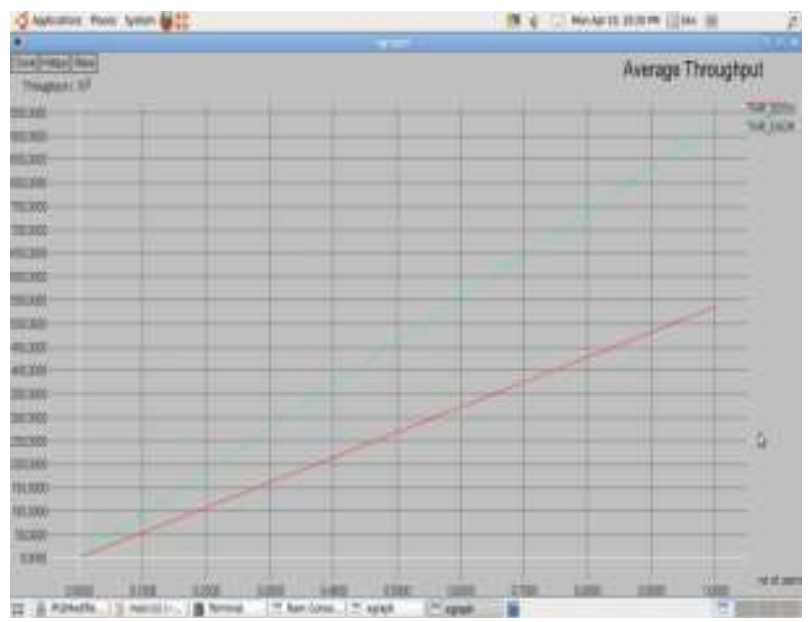

Energy Consumption

Bellow graph shows the comparison of existing and proposed scheme in terms of energy consumption. In this figure, the performance of proposed scheme is good and low energy usage level as compared to existing scheme.

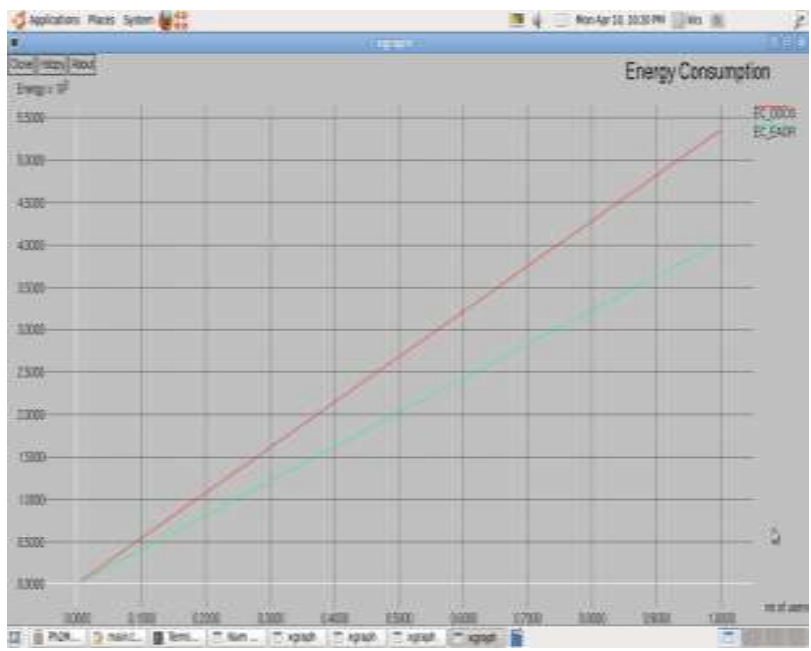

Packet Delivery Ratio

Bellow graph shows the comparison of existing and proposed scheme in terms of Packet delivery ratio. In this figure, the performance of proposed scheme is good and high data delivery level as compared to existing scheme.

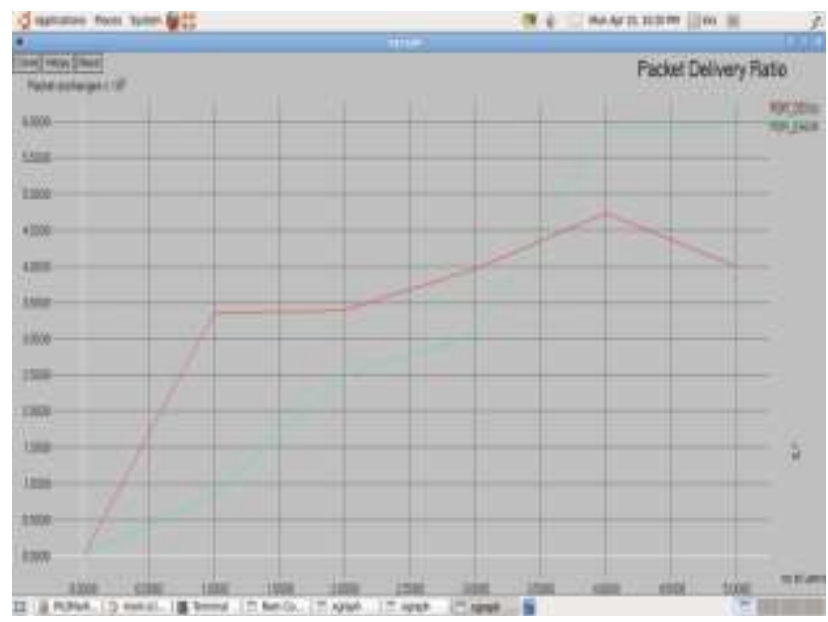




\section{CONCLUSION}

Node's movement stability, channel load, node congestion level and route expiry time are the important QoS metrics among several QoS parameters for providing an efficient, low overhead QoS support for anycast routing in MANETs. We proposed mobility and QoS based anycast routing in MANETs. The proposed work is simulated for various MANET network environments to validate its performance. From the simulations, we observed that the proposed scheme performs better than traditional flooding, DSR scheme in terms of control overhead, packet delivery ratio and end-to-end delay.

Simulation procedure for the proposed scheme is as follows. Generate ad hoc network with given number of nodes. Estimate neighbor stability based on self node movement stability and neighbor node movement stability. Compute link congestion factor based on channel congestion and buffer congestion factors. Compute LET. Update link data base at each node considering their neighbors. Initiate Route Discovery Process using RR, RP and RE, and accordingly update RIC. This frame work achieves high security, more energy efficiency, high packet delivery ratio, only less delay, comparing to previous frame works.

In future, improve this process security as digital signature security frame work and our proposed security scheme for centralized topology networks, so in future we improve this security using digital signature security technique for decentralized large level networks topologies. We would like to work on anycast routing protocols to check the efficiency under high throughput applications, e.g. multimedia applications by employing negotiation parameters in route request packet in finding nearest server through non congestion paths.

\section{REFERENCES}

[1] Y. Shi, Y. T. Hou, S. Kompella, and H. D. Sherali, "Maximizing capacity in multihop cognitive radio networks under the SINR model," IEEE Trans. Mobile Comput., vol. 10, no. 7, pp. 954-967, Jul. 2011.

[2] Y. Shi, Y. T. Hou, and H. D. Sherali, "Cross-layer optimization for data rate utility problem in UWB-based ad hoc networks," IEEE Trans. Mobile Comput., vol. 7, no. 6, pp. 764-777, Jun. 2008

[3] Y. Shi, Y. T. Hou, J. Liu, and S. Kompella, "Bridging the gap between protocol and physical models for wireless networks," IEEE Trans. Mobile Comput., vol. 12, no. 7, pp. 1404-1416, Jul. 2013.

[4] D. Feng, C. Jiang, G. Lim, L. J. Cimini, Jr., G. Feng, and G. Y. Li, “A survey of energy-efficient wireless communications,'” IEEE Commun. Surveys Tuts., vol. 15, no. 1, pp. 167-178, first quarter 2013.

[5] C. Isheden, Z. Chong, E. Jorswieck, and G. Fettweis, "Framework for link-level energy efficiency optimization with informed transmitter," IEEE Trans. Wireless Commun., vol. 11, no. 8, pp. 2946-2957, Aug. 2012.

[6] G. Miao, N. Himayat, and G. Y. Li, “Energy-efficient link adaptation in frequency-selective channels,'” IEEE Trans. Commun., vol. 58, no. 2, pp. 545-554, Feb. 2010.

[7] S. Gao, L. Qian, and D. Vaman, “Distributed energy efficient spectrum access in cognitive radio wireless ad hoc networks," IEEE Trans. Wireless Commun., vol. 8, no. 10, pp. 5202-5213, Oct. 2009.

[8] F. Meshkati, H. V. Poor, S. C. Schwartz, and N. B. Mandayam, "An energy-efficient approach to power control and receiver design in wireless data networks,'” IEEE Trans. Commun., vol. 53, no. 11, pp. 1885-1894, Nov. 2005.

[9] F. Meshkati, H. V. Poor, S. C. Schwartz, and R. V. Balan, "Energyefficient resource allocation in wireless networks with quality-of-service constraints," IEEE Trans. Commun., vol. 57, no. 11, pp. 3406-3414, Nov. 2009.

[10] C. U. Saraydar, N. B. Mandayam, and D. J. Goodman, "Efficient power control via pricing in wireless data networks,' IEEE Trans. Commun., vol. 50, no. 2, pp. 291-303, Feb. 2002.

[11] D. W. K. Ng, E. S. Lo, and R. Schober, "Energy-efficient resource allocation in OFDMA systems with large numbers of base station antennas," IEEE Trans. Wireless Commun., vol. 11, no. 9, pp. 3292-3304, Sep. 2012.

[12] D. W. K. Ng, E. S. Lo, and R. Schober, "Energy-efficient resource allocation in multi-cell OFDMA systems with limited backhaul capacity," IEEE Trans. Wireless Commun., vol. 11, no. 10, pp. 3618-3631, Oct. 2012.

[13] D. W. K. Ng, E. S. Lo, and R. Schober, "Wireless information and power transfer: Energy efficiency optimization in OFDMA systems," IEEE Trans. Wireless Commun., vol. 12, no. 12, pp. 6352-6370, Dec. 2013.

[14] K. T. K. Cheung, S. Yang, and L. Hanzo, “Achieving maximum energy-efficiency in multi-relay OFDMA cellular networks: A fractional programming approach," IEEE Trans. Commun., vol. 61, no. 8, pp. 2746-2757, Jul. 2013.

[15] Q. Zhao and L. Tong, "Energy efficiency of large-scale wireless networks: Proactive versus reactive networking," IEEE J. Sel. Areas Commun., vol. 23, no. 5, pp. 1100-1112, May 2005.

[16] J. Zhang, L. Fei, Q. Gao, and X.-H. Peng, "Energy-efficient multihop cooperative MISO transmission with optimal hop distance in wireless ad hoc networks,' IEEE Trans. Wireless Commun., vol. 10, no. 10, pp. 3426-3435, Oct. 2011

[17] D. Yue, G. Guillén-Gosálbez, and F. You, "Global optimization of large-scale mixed-integer linear fractional programming problems: A reformulation-linearization method and process scheduling applications," AIChE J., vol. 59, no. 11, pp. 4255-4272, 2013.

[18] E. B. Bajalinov, Linear-Fractional Programming: Theory, Methods, Applications and Software. New York, NY, USA: Springer, 2003.

[19] T. Clausen and P. Jacquet, Optimized Link State Routing Protocol (OLSR), document RFC 3626, IETF, Oct. 2003;. [Online]. Available: http://www.ietf.org/rfc/rfc3626.txt.

[20] D. Johnson, Y. Hu, and D. Maltz, The Dynamic Source Routing Protocol (DSR) for Mobile Ad Hoc Networks for IPv4, document RFC 4728, IETF, Oct. 2003. [Online]. Available: http://www.ietf.org/rfc/rfc4728.txt 